\title{
Cost Implications of Comorbidity for Autologous Stem Cell Transplantation in Elderly Patients with Multiple Myeloma Using SEER-Medicare
}

\author{
Gunjan L. Shah, ${ }^{1}$ Aaron Winn, ${ }^{2}$ Pei-Jung Lin, ${ }^{2}$ Andreas Klein, ${ }^{3}$ \\ Kellie A. Sprague, ${ }^{3}$ Hedy P. Smith, ${ }^{3}$ Rachel Buchsbaum, ${ }^{3}$ Joshua T. Cohen, ${ }^{2}$ \\ Kenneth B. Miller, ${ }^{3}$ Raymond Comenzo, ${ }^{3}$ and Susan K. Parsons ${ }^{3,4}$ \\ ${ }^{1}$ Adult Bone Marrow Transplantation Service, Department of Medicine, Memorial Sloan-Kettering Cancer Center, \\ 1275 York Box 298 Avenue, New York, NY 10065, USA \\ ${ }^{2}$ Center for the Evaluation of Value and Risk in Health, Institute for Clinical Research and Health Policy Studies, \\ Tufts Medical Center, 800 Washington St., Box 63, Boston, MA 02111, USA \\ ${ }^{3}$ Division of Hematology/Oncology, Department of Medicine, Tufts Medical Center, 800 Washington St., Box 245, \\ Boston, MA 02111, USA \\ ${ }^{4}$ Center for Health Solutions, Institute for Clinical Research and Health Policy Studies, Tufts Medical Center, \\ 800 Washington St., Box 345, Boston, MA 02111, USA \\ Correspondence should be addressed to Gunjan L. Shah; gunjanlshah@gmail.com
}

Received 29 July 2016; Accepted 28 September 2016

Academic Editor: Paolo De Fabritiis

Copyright (c) 2016 Gunjan L. Shah et al. This is an open access article distributed under the Creative Commons Attribution License, which permits unrestricted use, distribution, and reproduction in any medium, provided the original work is properly cited.

\begin{abstract}
Comorbidity is more common in older patients and can increase the cost of care by increasing toxicity. Using the SEER-Medicare database from 2000 to 2007, we examined the costs and life-year benefit of Auto-HSCT for MM patients over the age of 65 by evaluating the difference over time relative to comorbidity burden. One hundred ten patients had an Auto-HSCT in the early time period (2000-2003) and 160 in the late time period (2004-2007). Patients were divided by a Charlson Comorbidity Index (CCI) of 0 or greater than 1 (CCI1+). Median overall survival was 53.5 months for the late time period patients compared to 40.3 months for the early time period patients ( $p=0.031$ ). Median costs for CCI0 versus CCI1+ in the early period were, respectively, $\$ 70,900$ versus $\$ 72,000$ (100 d); $\$ 86,100$ versus $\$ 98,300$ ( $1 \mathrm{yr}$ ); and $\$ 139,200$ versus $\$ 195,300$ (3 yrs). Median costs for late period were, respectively, $\$ 58,400$ versus $\$ 60,400(100 \mathrm{~d}) ; \$ 86,300$ versus $\$ 77,700$ ( $1 \mathrm{yr})$; and $\$ 124,400$ versus $\$ 110,900$ ( 3 yrs). Comorbidity had a significant impact on survival and cost among early time period patients but not among late time period patients. Therefore, older patients with some comorbidities can be considered for Auto-HSCT depending on clinical circumstances.
\end{abstract}

\section{Introduction}

Medicare coverage of Auto-HSCT for MM began in 2000. By 2009 , patients over the age of 60 accounted for $40 \%$ of AutoHSCT procedures, with the leading indication being MM [1]. Older cancer patients are known to have more preexisting health conditions [2], and higher comorbidity assessment before transplant in MM is correlated with increased toxicity [3], worse survival [4], and longer length of stay [5]. The cost implications of the rise in Auto-HSCT for the elderly are not well described, with most studies focusing on younger patients at single institutions [6-10]. Using the Surveillance,
Epidemiology, and End Results- (SEER-) Medicare database from 2000 to 2007, we examined the costs and life-year benefit of Auto-HSCT for MM patients over the age of 65 by evaluating the difference over time relative to comorbidity burden.

\section{Patients and Methods}

2.1. Data Source. SEER registries include the Alaska Native Tumor Registry, Arizona Indians, Cherokee Nation, Connecticut, Detroit, Georgia (Atlanta, Greater Georgia, and 
Rural Georgia), California (Greater Bay Area Cancer Registry, Los Angeles, and Greater California), Hawaii, Iowa, Kentucky, Louisiana, New Jersey, New Mexico, Seattle-Puget Sound, and Utah. For each cancer diagnosis collected by a SEER registry, demographics and selected diagnostic and treatment information, along with date and cause of death, are collected. Currently, the SEER registries cover approximately $28 \%$ of the United States population and have an internal quality control process to ensure the accuracy of the data [11]. Among the patients who are at least 65 years old in the SEER registry, 93\% have been linked to their fee-forservice (FFS) Medicare claims $[12,13]$. The linkage completed in 2011 includes SEER incident cases through December 31, 2007, and Medicare claims through December 31, 2009. The Patient Entitlement and Diagnosis Summary (Medicare enrollment information), the Medicare Provider Analysis and Review (MEDPAR) (inpatient Medicare Part A claims), the National Claims History (NCH) file (provider Medicare Part B claims), and the Outpatient (institutional Medicare Part B claims) files, Durable Medical Equipment files, Hospice files, and Home Health files were used in this analysis. The study was approved by the Tufts Medical Center Institutional Review Board.

2.2. Patient Sample. We identified patients with $\mathrm{MM}$ in SEER by the International Classification of Disease-Oncology Version 3 (ICD-O-3) code, 9731-2, restricting our study to cases diagnosed after October 2000, when Medicare coverage of Auto-HSCT began. We used International Classification of Disease-9 (ICD-9, 41.00, 41.01, 41.04, 41.07, 41.09) or Healthcare Common Procedure Coding System (HCPCS, 38241) codes to identify patients as having an Auto-HSCT after MM diagnosis. We included only those cases enrolled in Medicare Part A and B FFS plans and limited attention to individuals over age 65 at diagnosis in order to calculate the comorbidity index. We then divided cases into an early time period (diagnosed October 1, 2000-December 31, 2003) and late time period (diagnosed January 1, 2004-December 31, 2007). We chose these dates to facilitate comparison of outcomes for patients before and after the introduction of bortezomib, which we hypothesized would add substantial cost compared to the previous therapeutic option of vincristine, doxorubicin, and dexamethasone. Bortezomib was FDA-approved in June 2003, with Center for Medicare Services (CMS) passthrough billing starting January 1, 2004 [14].

2.3. Comorbidity. We modified calculation of the original Charlson Comorbidity Index (CCI) using ICD-9 codes from Medicare claims in the year prior to MM diagnosis [15]. We divided cases into two groups: those individuals with a CCI of 0 (no comorbidities) and those with a CCI score of 1 or more (designated "1+"). This cutoff was chosen due to the small number of patients with more than one comorbidity (Table 1). In addition, as comorbidity can be underrepresented by billing data $[15,16]$ any comorbidity was considered significant.

2.4. Statistical Methods. All statistical analyses were performed using SAS (version 9.3, SAS, Cary, NC), Stata (version
12, StataCorp, College Station, TX), and Excel (Microsoft, Redmond, WA). Demographic characteristics were compared using two-sided $t$-tests and chi-squared tests.

We determined the date of death from the SEER Patient Entitlement and Diagnosis Summary File. We calculated survival for the first 100 days (d), 1 year (yr), and 3 yrs after diagnosis using Kaplan-Meier curves. We included up to 9 years of follow-up data and calculated the log rank test for comparison. Using the medical care component of the Consumer Price Index, we inflation-adjusted cost data, converting it to 2010 US dollars [17, 18]. We then calculated costs for the first 100 days (d), 1 year (yr), 3 yrs, and 5 yrs after diagnosis along with the cumulative cost since diagnosis. Cumulative per patient costs were calculated, averaged, and plotted to compare the total costs in the two time periods.

\section{Results}

3.1. Cohort Characteristics. Of the 22,286 patients in SEERMedicare who were diagnosed with MM between 2000 and 2007, 6,078 (27\%) were over the age of 65 and enrolled in Medicare Part A and B FFS. Of these, 270 (4.4\%) met our billing code criteria for having undergone an Auto-HSCT (110, early; 160, late). Characteristics between time periods did not differ statistically (Table 1). Forty percent of the subjects in each time period were female and most were Caucasian (88\%, early; $91 \%$, late). The median age in both time periods was 70 years (range: 66-92 yrs (early); range: $66-90$ yrs (late)). Only a small portion of patients was over 81 yrs (3\% versus $1 \%$ for the early and late time periods, resp.). Comorbidity did not differ over time. The CCI was 0 for $62 \%$ early time period subjects and $63 \%$ for late time period subjects.

3.2. Survival. The median time to transplant was significantly longer for late time period subjects $(277 \mathrm{~d})$ than for early period subjects $(223 \mathrm{~d})(p=0.03)$. More than $96 \%$ of transplants occurred within 3 years of diagnosis for patients in both time periods. While survival after Auto-HSCT in the early time was associated with comorbidity level, this pattern was not observed in the late time period (Table 1). Median overall survival was significantly longer for late time period patients (53.3 months) than for early time period patients (40.3 months) ( $p=0.031$, Figure 1$)$. The subset of patients over the age of 70 had a 5 -year OS of $25 \%$ in the early time period compared to $65 \%$ for the late group.

3.3. Cost. As with survival, higher comorbidity scores were associated with an increased cost among early period patients, whereas, for late period patients, costs did not differ statistically between CCI0 and CCI1+ patients (Table 1). Median costs for CCI0 versus CCI1+ in the early period were, respectively, $\$ 70,900$ versus $\$ 72,000$ (100 d); $\$ 86,100$ versus $\$ 98,300$ (1 yr); and $\$ 139,200$ versus $\$ 195,300$ (3 yrs). Median costs for the late period were, respectively, $\$ 58,400$ versus $\$ 60,400$ (100 d); $\$ 86,300$ versus $\$ 77,700$ (1 yr); and $\$ 124,400$ versus $\$ 110,900$ (3 yrs). In addition, the average per person cumulative cost remained lower for late period patients for at least six years after transplant (Figure 2). Finally, using 
TABLE 1: Patient characteristics. Early transplants are those from 2000 to 2003, while late transplants are between 2004 and 2007.

\begin{tabular}{|c|c|c|c|c|}
\hline & \multicolumn{2}{|c|}{ Early } & \multicolumn{2}{|c|}{ Late } \\
\hline Patient characteristics & \multicolumn{2}{|c|}{$N=110,(\%)$} & \multicolumn{2}{|c|}{$N=160,(\%)$} \\
\hline Female & \multicolumn{2}{|c|}{$45(41)$} & \multicolumn{2}{|c|}{$64(40)$} \\
\hline White & \multicolumn{2}{|c|}{$97(88)$} & \multicolumn{2}{|c|}{$145(91)$} \\
\hline Age, median (range) & \multicolumn{2}{|c|}{$70(66-92)$} & \multicolumn{2}{|c|}{$70(66-90)$} \\
\hline $66-70$ & \multicolumn{2}{|c|}{$75(68)$} & \multicolumn{2}{|c|}{$100(63)$} \\
\hline $70-74$ & \multicolumn{2}{|c|}{$21(19)$} & \multicolumn{2}{|c|}{$47(29)$} \\
\hline $75+$ & \multicolumn{2}{|c|}{$14(13)$} & \multicolumn{2}{|c|}{$13(8)$} \\
\hline \multicolumn{5}{|c|}{ Charlson Comorbidity Index } \\
\hline 0 & \multicolumn{2}{|c|}{$68(62)$} & \multicolumn{2}{|c|}{$101(63)$} \\
\hline 1 & \multicolumn{2}{|c|}{$29(26)$} & \multicolumn{2}{|c|}{$37(23)$} \\
\hline $2+$ & \multicolumn{2}{|c|}{$13(12)$} & \multicolumn{2}{|c|}{$22(14)$} \\
\hline Survival outcomes & CCIO & CCI1+ & CCIO & CCI1+ \\
\hline 100 days $^{*}$ & $95 \%$ & $85 \%$ & $99 \%$ & $93 \%$ \\
\hline 1 year** $^{* *}$ & $90 \%$ & $76 \%$ & $92 \%$ & $86 \%$ \\
\hline 3 years ${ }^{* * *}$ & $62 \%$ & $42 \%$ & $66 \%$ & $66 \%$ \\
\hline $\begin{array}{l}\text { Cost outcomes } \\
\text { Median }(25 \% ; 75 \%)\end{array}$ & CCI0 & CCI1+ & CCI0 & CCI1+ \\
\hline 100 days & $\begin{array}{c}N=65 \\
\$ 70,900 \\
(46,000 ; 85,200)\end{array}$ & $\begin{array}{c}N=36 \\
\$ 72,000 \\
(55,600 ; 81,100)\end{array}$ & $\begin{array}{c}N=100 \\
\$ 58,400 \\
(37,900 ; 73,900)\end{array}$ & $\begin{array}{c}N=54 \\
\$ 60,400 \\
(50,300 ; 70,100)\end{array}$ \\
\hline 1 year & $\begin{array}{c}N=61 \\
\$ 86,100 \\
(59,500 ; 113,100)\end{array}$ & $\begin{array}{c}N=31 \\
\$ 98,300 \\
(75,100 ; 116,700)\end{array}$ & $\begin{array}{c}N=93 \\
\$ 81,300 \\
(59,400 ; 112,300)\end{array}$ & $\begin{array}{c}N=49 \\
\$ 77,700 \\
(69,300 ; 91,800)\end{array}$ \\
\hline 3 years & $\begin{array}{c}N=42 \\
\$ 139,200 \\
(94,200 ; 179,900)\end{array}$ & $\begin{array}{c}N=17 \\
\$ 195,300 \\
(153,300 ; 224,600)\end{array}$ & $\begin{array}{c}N=45 \\
\$ 124,400 \\
(97,300 ; 159,500)\end{array}$ & $\begin{array}{c}N=29 \\
\$ 110,900 \\
(89,600 ; 167,300)\end{array}$ \\
\hline
\end{tabular}

${ }^{*}$ Early $p=0.06$, late $p=0.098 .{ }^{* *}$ Early $p=0.043$, late $p=0.20 .{ }^{* * *}$ Early $p=0.34$, late $p=0.86$.

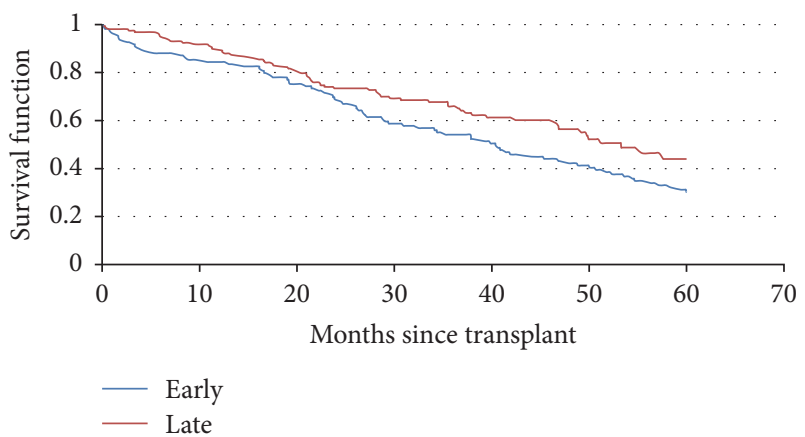

FIgURE 1: Overall survival after transplant. Early transplants are those from 2000 to 2003, while late transplants are between 2004 and 2007. Median overall survival for late time period patients was 53.3 months and for early time period patients was 40.3 months $(p=0.031)$.

quantile regression, stratifying by age $(>70$ versus $\leq 70)$ and comorbidity did not change the cost in either the early or late time period.

\section{Discussion}

Although recent advances in the management of MM have enhanced responses $[19,20]$ and extended survival $[21,22]$

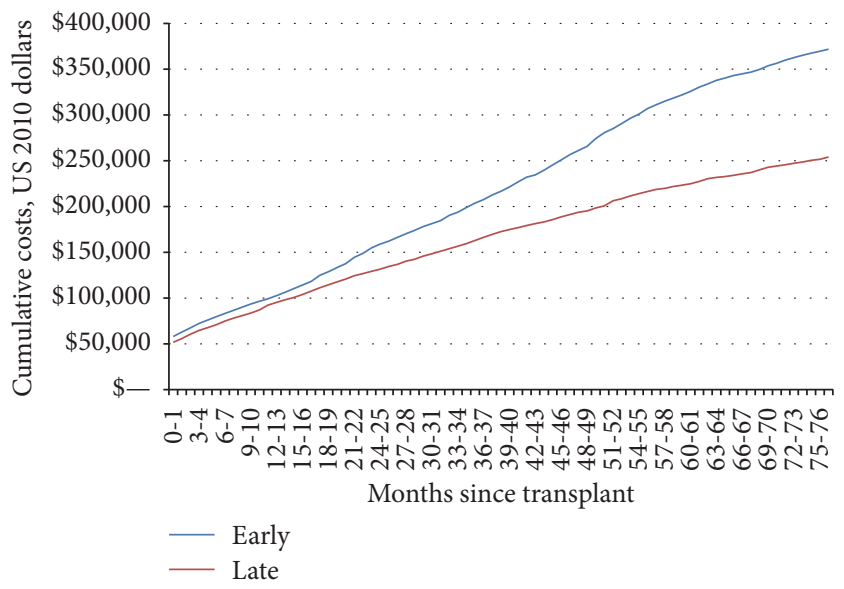

FIGURE 2: Average cumulative per person cost. Early transplants are those from 2000 to 2003, while late transplants are between 2004 and 2007. The cumulative cost was lower for patients transplant in the later time period compared to the earlier.

the cost of these advancements has not been evaluated in elderly patients. As contemporary therapy has allowed a greater percentage of older patients to become transplant eligible, the impact of comorbidities upon survival must be 
taken into account. In a recent study by Saad et al., the application of the hematopoietic cell transplant comorbidity index (HCT-CI) to Auto-HSCT revealed that higher HCTCI correlated with inferior survival, but not with nonrelapse mortality [4]. Studies have reported both positive [23,24] and negative [25-27] correlations between cost and comorbidity in patients with solid tumors, but similar studies have not been conducted in patients with hematologic malignancies.

This is the first paper to compare the costs of AutoHSCT and comorbidity in elderly patients with MM in the era of novel agents, notably bortezomib. Our findings showed that comorbidities were significantly associated with higher health care costs in the early time period, but not afterwards. The lack of association between cost and comorbidity in the late period may be the result of improved responses with less pre-Auto-HSCT therapy. In particular, patients undergoing Auto-HSCT in the later time period may have experienced lower rates of treatment-induced toxicity secondary to comorbidities.

Our results, demonstrating lower 100-day survival rates than previously published $[28,29]$, may be due to the exclusion of high volume centers in SEER or the inclusion of older patients. However, Majhail et al. found, using Thomson Reuters MarketScan data, that $8 \%$ of MM and nonHodgkin's lymphoma patients over age 60 disenrolled from private insurance within 100 days of Auto-HSCT, which they considered a surrogate for mortality [30]. The Majhail et al. results are comparable to those with CCI1+ in the later time period. One-year survival in the more recent time frame for those with no comorbidities is also consistent with the recently published Center for International Blood and Marrow Transplant Research (CIBMTR) analysis [31]. Threeyear survival rates in the late period are also comparable to survival reported by the CIBMTR [1] and a meta-analysis of three international trials [32]. The improvement is likely related to better supportive care [33] and improved response rates with novel agents [22].

First 100-day costs in our study ranged from medians of $\$ 58,400$ to $\$ 78,000$ depending on time period and comorbidity. In a study looking at a national private claims database, median 100-day costs were $\$ 90,000$ for MM patients [30]. Furthermore, they found that median costs were lower for ages 41-60yrs than for ages 21-40 yrs, which mirror our results. Comparison to other studies with younger patients is difficult given that most occur outside the US, did not include novel agents, and did not evaluate comorbidity $[6,10,34,35]$. However, the costs in our study are similar to those found in a study of the Nationwide Inpatient Sample where the mean age was 55 years [36].

We acknowledge our study's limitations. First, because Medicare Part D prescription coverage for oral prescriptions did not begin until 2009, costs for oral medication (primarily lenalidomide and thalidomide, FDA-approved in 2006) were not included in our analysis. Future studies will be needed to address the contribution of oral therapies to the overall cost of care. In contrast, costs associated with bortezomib use are included. Second, claims databases often underrepresent comorbidity [15], although this issue may not have biased our analysis since the information would presumably be missing from both groups we compared (patients from the early and late time periods). Finally, because SEER does not collect the laboratory values, disease burden, or risk classification for MM, we cannot compare treatment response or diseasefree survival between the two time periods. Inclusions of Durie Salmon staging was initiated only in 2011 and the International Staging System is still not captured in SEER [11, 37-40]. Further evaluation of clinical trial or registrybased data may help to provide specific circumstances in which Auto-HSCT may be more or less cost effective, based on prognostic factors or response to initial therapy.

Despite these limitations, the strength of this analysis is the use of a nationally representative sample of patients, allowing for generalizability of our findings [41]. The link of SEER registry data (i.e., diagnosis, date of diagnosis, and updated survival information) with comprehensive cost date eliminates many of the shortcomings of other administrative databases, such as loss of coverage due to insurance churning, or the use of single institutional experience. The use of Medicare data allows capture of longitudinal claims from diagnosis until death in any state or institution where the interventions took place.

\section{Conclusion}

Overall, we found that the median time to transplant was significantly longer in late time period patients. Comorbidity was significantly associated with worse survival and higher health care costs in early time period patients, but not the late time period patients. Finally, with a similar comorbidity distribution over time, late time period patients had significantly extended survival and lower cumulative costs, compared to early time period patients.

\section{Disclosure}

Poster presentation was at the 55th annual meeting of the American Society of Hematology, New Orleans, LA, December 7, 2013, Abstract \#1746. The content is solely the responsibility of the authors and does not necessarily represent the official views of the National Institutes of Health.

\section{Competing Interests}

The authors declare no competing financial interests.

\section{Authors' Contributions}

Gunjan L. Shah, Andreas Klein, and Susan K. Parsons conceived and designed the study. Gunjan L. Shah, Aaron Winn, Pei-Jung Lin, Andreas Klein, Rachel Buchsbaum, Joshua T. Cohen, and Susan K. Parsons analyzed and interpreted the data. Gunjan L. Shah drafted the manuscript, and Aaron Winn, Pei-Jung Lin, Andreas Klein, Kellie A. Sprague, Hedy P. Smith, Rachel Buchsbaum, Joshua T. Cohen, Kenneth B. Miller, Raymond Comenzo, and Susan K. Parsons critically reviewed it. 


\section{Acknowledgments}

Research reported in this publication was supported by the National Cancer Institute of the National Institutes of Health under Award no. T32 CA009429 (GLS).

\section{References}

[1] M. C. Pasquini and Z. Wang, "Current use and outcome of hematopoietic stem cell transplantation: CIBMTR Summary Slides, 2013," http://www.cibmtr.org.

[2] J. F. Piccirillo, A. Vlahiotis, L. B. Barrett, K. L. Flood, E. L. Spitznagel, and E. W. Steyerberg, "The changing prevalence of comorbidity across the age spectrum," Critical Reviews in Oncology/Hematology, vol. 67, no. 2, pp. 124-132, 2008.

[3] L. Labonté, T. Iqbal, M. A. Zaidi et al., "Utility of comorbidity assessment in predicting transplantation-related toxicity following autologous hematopoietic stem cell transplantation for multiple myeloma," Biology of Blood and Marrow Transplantation, vol. 14, no. 9, pp. 1039-1044, 2008.

[4] A. Saad, A. Mahindra, M.-J. Zhang et al., "Hematopoietic cell transplant comorbidity index is predictive of survival after autologous hematopoietic cell transplantation in multiple myeloma," Biology of Blood and Marrow Transplantation, vol. 20, no. 3, pp. 402-408.el, 2014.

[5] M. Kleber, G. Ihorst, M. Terhorst et al., "Comorbidity as a prognostic variable in multiple myeloma: comparative evaluation of common comorbidity scores and use of a novel MMcomorbidity score," Blood Cancer Journal, vol. 1, no. 9, article e35, 2011.

[6] I. Buijt, G. J. Ossenkoppele, C. A. Uyl-De Groot, and P. C. Huijgens, "Costs of intensive treatment and follow-up of patients with multiple myeloma," Anti-Cancer Drugs, vol. 9, no. 10, pp. 889-897, 1998.

[7] C. Pandya, S. Hashmi, N. Khera et al., "Cost-effectiveness analysis of early vs. late autologous stem cell transplantation in multiple myeloma," Clinical transplantation, vol. 28, no. 10, pp. 1084-1091, 2014.

[8] S. Jagannath, D. H. Vesole, M. Zhang et al., "Feasibility and cost-effectiveness of outpatient autotransplants in multiple myeloma," Bone Marrow Transplantation, vol. 20, no. 6, pp. 445450, 1997.

[9] C. T. Kouroukis, B. J. O’Brien, A. Benger et al., "Cost-effectiveness of a transplantation strategy compared to melphalan and prednisone in younger patients with multiple myeloma," Leukemia and Lymphoma, vol. 44, no. 1, pp. 29-37, 2003.

[10] K. Moeremans and L. Annemans, "An update: health economics of managing multiple myeloma," European Journal of Cancer, vol. 42, no. 11, pp. 1684-1691, 2006.

[11] "About the SEER Program," Surveillance Research Program, NCI, http://seer.cancer.gov/about/overview.html.

[12] A.-M. Noone, J. L. Lund, A. Mariotto et al., "Comparison of SEER treatment data with medicare claims," Medical Care, vol. 54, no. 9, pp. e55-e64, 2016.

[13] A. Potosky, G. Riley, and J. Lubitz, "Potential for cancer related health services research using a linked Medicare-tumor registry database," Medical Care, vol. 31, pp. 732-748, 1993.

[14] "CMS Manual System Pub. 100-20 One-Time Notification," 2003.

[15] C. N. Klabunde, A. L. Potosky, J. M. Legler, and J. L. Warren, "Development of a comorbidity index using physician claims data," Journal of Clinical Epidemiology, vol. 53, no. 12, pp. 1258 1267,2000

[16] J. H. Garvin, A. Redd, D. Bolton et al., "Exploration of ICD-9CM coding of chronic disease within the Elixhauser Comorbidity Measure in patients with chronic heart failure," Perspectives in Health Information Management, vol. 10, p. 1, 2013.

[17] J. Newhouse, "Medical Care Price Indices: Problems and Opportunities," 2001.

[18] "U.S. Bureau of Labor Statistics," http://www.bls.gov/home .htm.

[19] J.-L. Harousseau, M. Attal, H. Avet-Loiseau et al., "Bortezomib plus dexamethasone is superior to vincristine plus doxorubicin plus dexamethasone as induction treatment prior to autologous stem-cell transplantation in newly diagnosed multiple myeloma: results of the IFM 2005-01 phase III trial," Journal of Clinical Oncology, vol. 28, no. 30, pp. 4621-4629, 2010.

[20] P. G. Richardson, E. Weller, S. Lonial et al., "Lenalidomide, bortezomib, and dexamethasone combination therapy in patients with newly diagnosed multiple myeloma," Blood, vol. 116, no. 5, pp. 679-686, 2010

[21] S. K. Kumar, S. V. Rajkumar, A. Dispenzieri et al., "Improved survival in multiple myeloma and the impact of novel therapies," Blood, vol. 111, no. 5, pp. 2516-2520, 2008.

[22] S. Ozaki, T. Harada, T. Saitoh et al., "Survival of multiple myeloma patients aged 65-70 years in the era of novel agents and autologous stem cell transplantation," Acta Haematologica, vol. 132, no. 2, pp. 211-219, 2014

[23] A. Woldemichael, E. Onukwugha, Z. Zheng, N. Hanna, B. S. Seal, and C. D. Mullins, "The impact of comorbidity on costs and effects of second-line treatment among elderly metastatic colon cancer patients," Journal of Clinical Oncology, vol. 32, supplement 3, abstract 536, 2014.

[24] D. J. Genther and C. G. Gourin, "Effect of comorbidity on shortterm outcomes and cost of care after head and neck cancer surgery in the elderly," Head and Neck, vol. 37, no. 5, pp. 685693,2015

[25] S. H. Taplin, W. Barlow, N. Urban et al., "Stage, age, comorbidity, and direct costs of colon, prostate, and breast cancer care," Journal of the National Cancer Institute, vol. 87, no. 6, pp. 417426, 1995.

[26] C. S. Hollenbeak, B. C. Stack Jr., S. M. Daley, and J. F. Piccirillo, "Using comorbidity indexes to predict costs for head and neck cancer," Archives of Otolaryngology-Head and Neck Surgery, vol. 133, no. 1, pp. 24-27, 2007.

[27] A. M. Bell, M. Corral, J. R. Penrod et al., "New drugs not major cost drivers for lung cancer care," American Journal of Pharmacy Benefits, vol. 6, no. 3, pp. e50-e59, 2014.

[28] M. A. Gertz, S. M. Ansell, D. Dingli et al., "Autologous stem cell transplant in 716 patients with multiple myeloma: low treatment-related mortality, feasibility of outpatient transplant, and effect of a multidisciplinary quality initiative," Mayo Clinic Proceedings, vol. 83, no. 10, pp. 1131-1135, 2008.

[29] D. E. Reece, C. Bredeson, W. S. Pérez et al., "Autologous stem cell transplantation in multiple myeloma patients $<60$ vs $\geq 60$ years of age," Bone Marrow Transplant, vol. 32, no. 12, pp. 1135-1143, 2003.

[30] N. S. Majhail, L. W. Mau, E. M. Denzen, and T. J. Arneson, "Costs of autologous and allogeneic hematopoietic cell transplantation in the United States: a study using a large National Private Claims Database," Bone Marrow Transplantation, vol. 48, no. 2, pp. 294-300, 2013. 
[31] M. Sharma, M.-J. Zhang, X. Zhong et al., "Older patients with myeloma derive similar benefit from autologous transplantation," Biology of Blood and Marrow Transplantation, vol. 20, no. 11, pp. 1796-1803, 2014.

[32] P. Sonneveld, H. Goldschmidt, L. Rosiñol et al., "Bortezomibbased versus nonbortezomib-based induction treatment before autologous stem-cell transplantation in patients with previously untreated multiple myeloma: a meta-analysis of phase III randomized, controlled trials," Journal of Clinical Oncology, vol. 31, no. 26, pp. 3279-3287, 2013.

[33] P. L. McCarthy, T. Hahn, A. Hassebroek et al., "Trends in use of and survival after autologous hematopoietic cell transplantation in North America, 1995-2005: significant improvement in survival for lymphoma and myeloma during a period of increasing recipient age," Biology of Blood and Marrow Transplantation, vol. 19, no. 7, pp. 1116-1123, 2013.

[34] V. Mishra, S. Andresen, L. Brinch et al., "Cost of autologous peripheral blood stem cell transplantation: the Norwegian experience from a multicenter cost study," Bone Marrow Transplantation, vol. 35, no. 12, pp. 1149-1153, 2005.

[35] M. Van Agthoven, C. M. Segeren, I. Buijt et al., "A cost-utility analysis comparing intensive chemotherapy alone to intensive chemotherapy followed by myeloablative chemotherapy with autologous stem-cell rescue in newly diagnosed patients with stage II/III multiple myeloma: A Prospective Randomised Phase III Study," European Journal of Cancer, vol. 40, no. 8, pp. 1159-1169, 2004.

[36] J. A. Jones, M. H. Qazilbash, Y.-C. T. Shih, S. B. Cantor, C. D. Cooksley, and L. S. Elting, "In-hospital complications of autologous hematopoietic stem cell transplantation for lymphoid malignancies: clinical and economic outcomes from the Nationwide Inpatient Sample," Cancer, vol. 112, no. 5, pp. 10961105, 2008.

[37] "Measures that are limited or not available in the data," NCI, http://appliedresearch.cancer.gov/seermedicare/considerations/measures.html.

[38] J. J. Young, S. Roffers, L. Ries, and A. Fritz, EER Summary Staging Manual-2000: Codes and Coding Instructions, Edited by Hurlbut, National Cancer Institute, Bethesda, Md, USA, 2001.

[39] J. Ruhl, M. Adamo, L. Dickie, L. Sun, and C. Johnson, Hematopoietic and Lymphoid Neoplasm Coding Manual, National Cancer Institute, Bethesda, Md, USA, 2014.

[40] Require SEER Site Specific Factors for Collaborative Stage, National Cancer Institute, Bethesda, Md, USA, 2014, http://seer .cancer.gov/tools/ssf/.

[41] J. L. Warren, C. N. Klabunde, D. Schrag, P. B. Bach, and G. F. Riley, "Overview of the SEER-Medicare data: content, research applications, and generalizability to the United States elderly population," Medical Care, vol. 40, no. 8, supplement, pp. IV3-IV-18, 2002. 


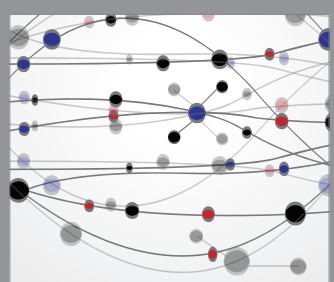

The Scientific World Journal
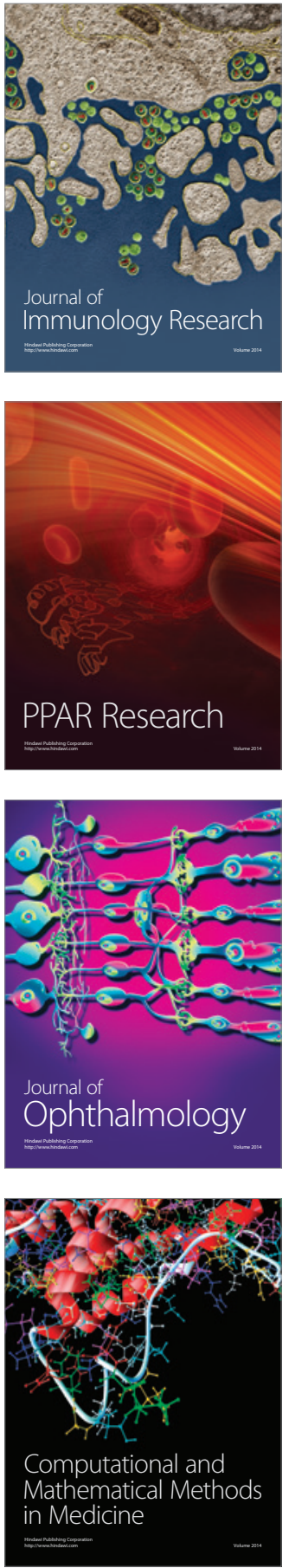

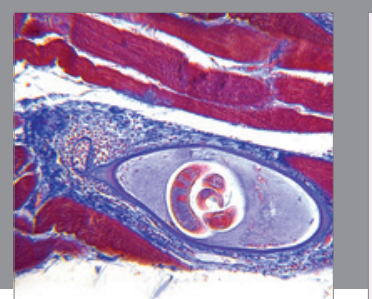

Gastroenterology Research and Practice

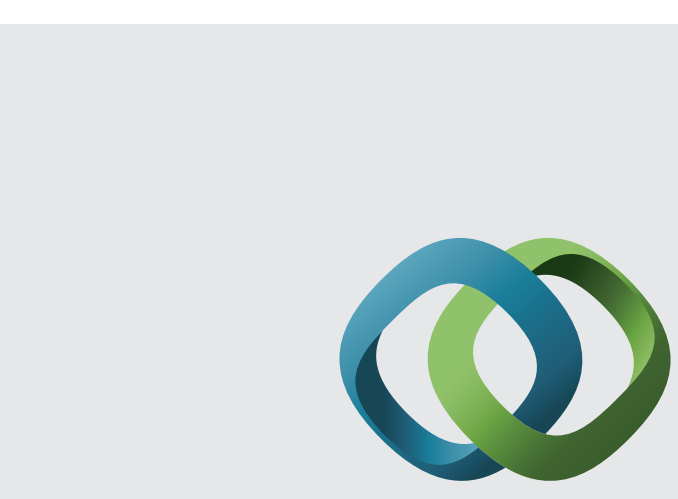

\section{Hindawi}

Submit your manuscripts at

http://www.hindawi.com
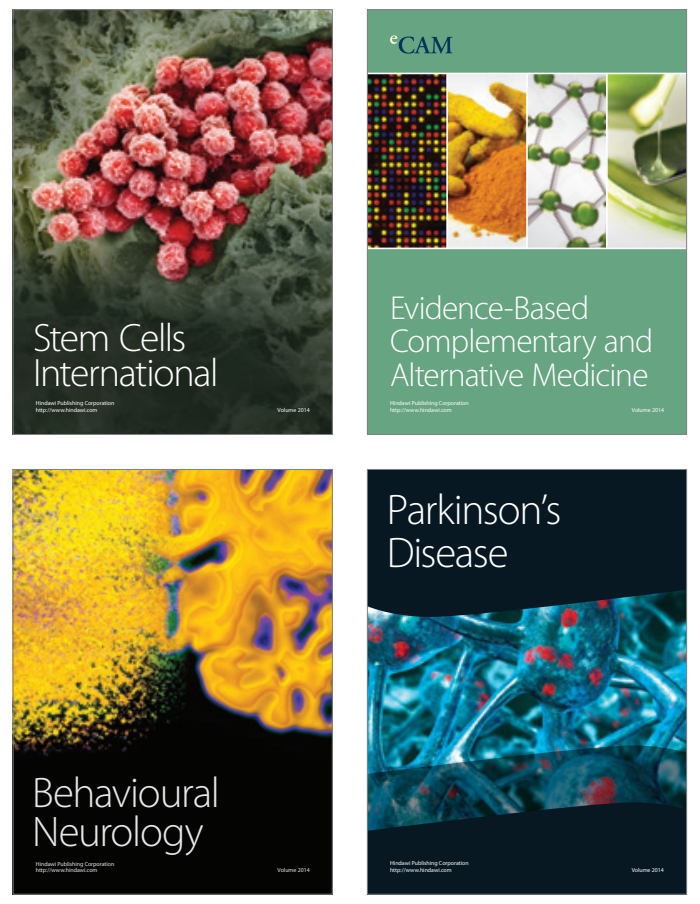
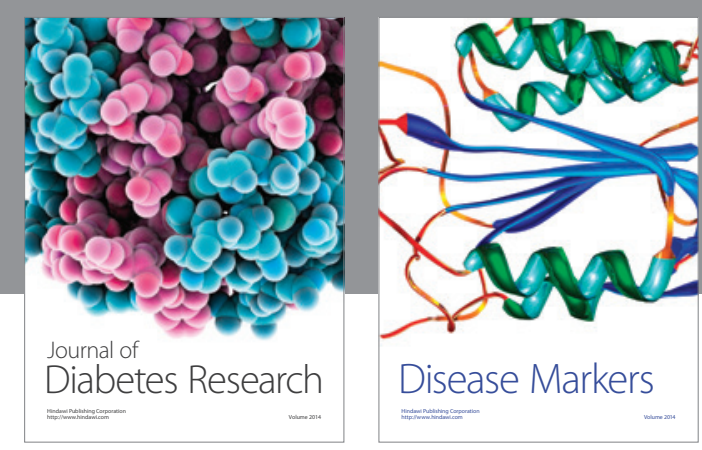

Disease Markers
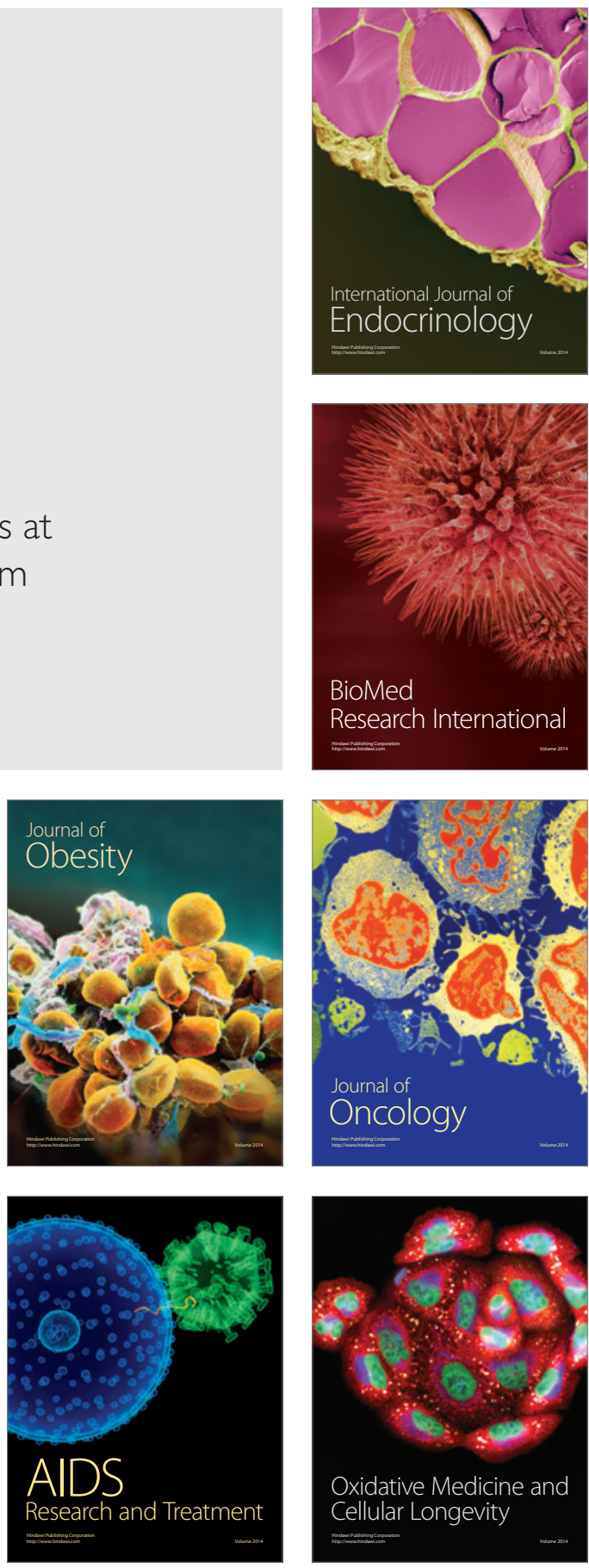